\title{
Intense monochromatic terahertz electromagnetic waves from coherent GaAs-like longitudinal optical phonons in (11n)-oriented GaAs/In0.1Al0.9As strained multiple quantum wells
}

Hideo Takeuchi, Souta Asai, Syuichi Tsuruta, and

Masaaki Nakayama

\begin{tabular}{|c|l|}
\hline Citation & Applied Physics Letters, 100(24): 242107 \\
\hline Issue Date & $2012-06$-15 \\
\hline Type & Journal Article \\
\hline Textversion & publisher \\
\hline Right & $\begin{array}{l}\text { A } 2012 \text { American Institute of Physics. This article may be downloaded for personal use only. } \\
\text { The following article appeared in Applied Physics Letters and maybe found at } \\
\text { https://doi.org/10.1063/1.4729125 }\end{array}$ \\
\hline DOI & $10.1063 / 1.4729125$ \\
\hline
\end{tabular}

Self-Archiving by Author(s)

Placed on: Osaka City University Repository

TAKEUCHI, H., ASAI, S., TSURUTA, S., \& NAKAYAMA, M. (2012). Intense monochromatic terahertz electromagnetic waves from coherent GaAs-like longitudinal optical phonons in (11n)-oriented GaAs/In 0 . 1Al 0 . 9As strained multiple quantum wells (4 pages). APPLIED PHYSICS LETTERS. 100, 242107. 


\section{AIP Appinied Physics \\ Letters}

Intense monochromatic terahertz electromagnetic waves from coherent GaAs-like longitudinal optical phonons in (11n)-oriented GaAs/In0.1Al0.9As strained multiple quantum wells

Hideo Takeuchi, Souta Asai, Syuichi Tsuruta, and Masaaki Nakayama

Citation: Appl. Phys. Lett. 100, 242107 (2012); doi: 10.1063/1.4729125

View online: http://dx.doi.org/10.1063/1.4729125

View Table of Contents: http://apl.aip.org/resource/1/APPLAB/v100/i24

Published by the American Institute of Physics.

\section{Related Articles}

Optically addressed near and long-wave infrared multiband photodetectors

Appl. Phys. Lett. 100, 241103 (2012)

InGaN/GaN single-quantum-well microdisks

Appl. Phys. Lett. 100, 242101 (2012)

Increasing the optical transition oscillator strength in GaSb-based type II quantum wells

Appl. Phys. Lett. 100, 231908 (2012)

Characterization of low temperature InGaAs-InAIAs semiconductor photo mixers at $1.55 \mu \mathrm{m}$ wavelength illumination for terahertz generation and detection

J. Appl. Phys. 111, 103105 (2012)

A simple analysis of interband absorption in quantum well structure of III-V ternary and quaternary semiconductors

J. Appl. Phys. 111, 103104 (2012)

Additional information on Appl. Phys. Lett.

Journal Homepage: http://apl.aip.org/

Journal Information: http://apl.aip.org/about/about_the_journal

Top downloads: http://apl.aip.org/features/most_downloaded

Information for Authors: http://apl.aip.org/authors

\section{ADVERTISEMENT}

\section{Agilent Technologies}

Agilent Education and Research Resources DVD 2012

Packed with over 100 NEW articles, application notes, webcasts, and videos relating to Renewable Energy, Nanoscience, RF/Wireless, MIMO, Materials, Digital Signals, Photonics, and General Test \& Measurement.

\section{Click Here to

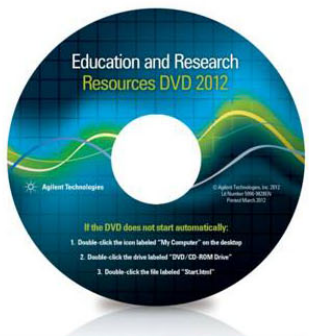




\title{
Intense monochromatic terahertz electromagnetic waves from coherent GaAs-like longitudinal optical phonons in (11n)-oriented GaAs/ $/ \mathrm{n}_{0.1} \mathrm{Al} \mathrm{I}_{0.9} \mathrm{As}$ strained multiple quantum wells
}

\author{
Hideo Takeuchi, ${ }^{1}$ Souta Asai, ${ }^{2}$ Syuichi Tsuruta $^{2}$ and Masaaki Nakayama ${ }^{2}$ \\ ${ }^{1}$ Department of Electronic Systems Engineering, School of Engineering, The University of Shiga Prefecture, \\ 2500 Hassaka-cho, Hikone, Shiga 522-8533, Japan \\ ${ }^{2}$ Department of Applied Physics, Graduate School of Engineering, Osaka City University, 3-3-138 Sugimoto, \\ Sumiyoshi-ku, Osaka 558-8585, Japan
}

(Received 31 March 2012; accepted 26 May 2012; published online 15 June 2012)

\begin{abstract}
We demonstrate that, in (11n)-oriented $\mathrm{GaAs} / \mathrm{In}_{0.1} \mathrm{Al}_{0.9} \mathrm{As}$ strained multiple quantum wells, the terahertz electromagnetic wave from the coherent GaAs-like longitudinal optical (LO) phonon is enhanced by a piezoelectric field originating from a tensile strain in the GaAs layer. The presence of the tensile strain is confirmed using Raman scattering spectroscopy. The Fourier power spectrum of the terahertz waveform shows that the intensity of the terahertz band of the coherent GaAs-like LO phonon increases as the index $n$ approaches 1 . The amplitude of the GaAs-like LO phonon is proportional to the piezoelectric field in the strained GaAs layer. (C) 2012 American Institute of Physics. [http://dx.doi.org/10.1063/1.4729125]
\end{abstract}

Intense monochromatic terahertz electromagnetic waves have been an attracting issue in the research field of the terahertz waves. ${ }^{1}$ Coherent optical phonons are characterized by oscillating polarization. Accordingly, the coherent optical phonon has a potential to emit a monochromatic terahertz wave. Historically, in the initial stage, bulk semiconductor crystals were applied to the terahertz emitters utilizing the coherent optical phonons; however, the emission intensity of the terahertz wave from the coherent optical phonon is typically low. ${ }^{2-8}$ The major reason is attributed to the fact that the dynamical dipole moments resulting from the coherent optical phonons are canceled by the translational symmetry of the lattice. This means that only the surface region contributes the terahertz emission because the lattice symmetry is broken in the surface region. In contrast to the bulk crystals, recently, Mizoguchi and Nakayama et al..$^{9-11}$ reported that the intense terahertz waves are emitted from GaAs-like longitudinal optical (LO) phonons confined in the GaAs layer in (001)-oriented GaAs/AlAs multiple quantum wells (MQWs). The intense emission of the terahertz wave originates from the fact that the confinement of the LO phonons corresponds to the symmetry breaking at each GaAs/AlAs interface. In the earlier works, only the (001)-oriented MQWs were used as samples for terahertz emitters. Here, we focus on the advantage of using GaAs-based strained MQWs with a $(11 n)$ orientation because the GaAs layer under an orthorhombic biaxial strain produces a piezoelectric field along the $[11 n]$ direction. The presence of the piezoelectric field $(\mathbf{E})$ results in the enhancement of the polarization $(\mathbf{P})$ of the LO phonon along the direction of the electric field, which is well established as the following equation: $\mathbf{P}=[\chi] \mathbf{E}$, where $[\chi]$ is an electric susceptibility tensor. The enhancement of the polarization connects with the enlargement of the initial displacement of the LO phonon. The initial displacement is released by an instantaneous change of the piezoelectric field by the screening effect due to photogenerated carriers, which launches the coherent oscillation of the LO phonon, i.e., the coherent LO phonon. ${ }^{12}$ This phenomenon leads to the oscillation of the LO-phonon polarization producing the terahertz wave. It is noted that the enlargement of the initial displacement results in the enhancement of the amplitude of the coherent LO phonon. Consequently, the presence of the piezoelectric field leads to the enhancement of the terahertz-wave intensity from the coherent LO phonon. Thus, the GaAs-based strained MQWs with the (11n) orientation give an additional degree of freedom to the strategy for enhancing the monochromatic terahertz wave from the coherent optical phonons.

In the present paper, we demonstrate that (11n)-oriented $\mathrm{GaAs} / \mathrm{In}_{0.1} \mathrm{Al}_{0.9} \mathrm{As}$ strained MQWs emit the intense terahertz waves from the coherent LO phonons at room temperature. We used Raman scattering spectroscopy in order to characterize the presence of biaxial tensile strains. We observed a strain-induced frequency shift of the GaAs-like LO phonon. The result of the Raman scattering measurement ensures that the GaAs layers are actually under the biaxial tensile strain in the (11n)-oriented strained MQWs. We measured the terahertz waves from the $(11 n)$-oriented strained MQWs in time domain. We found that the amplitude of the terahertz wave from the coherent GaAs-like LO phonon increases as the orientation index $n$ approaches 1 . The Fourier transform spectra of the terahertz waveforms show an intense monochromatic terahertz band resulting from the coherent GaAs-like LO phonon. The origin of the intense monochromatic terahertz band is discussed in terms of the effects of the piezoelectric field peculiar to the (11n)-oriented strained MQWs.

The present samples, the $\mathrm{GaAs} / \mathrm{In}_{0.1} \mathrm{Al}_{0.9} \mathrm{As}$ strained MQWs, were grown on (11n)-oriented semi-insulating GaAs substrates by molecular beam epitaxy. The orientation index $n$ took values of $2,3,4$, and $\infty$, where the value of $\infty$ corresponds to the (001) orientation. In advance to the growth of the MQW layer, an $\operatorname{In}_{0.1} \mathrm{Al}_{0.9} \mathrm{As}$ buffer layer with a thickness of $1.0 \mu \mathrm{m}$ was grown in each sample. The thickness of $1.0 \mu \mathrm{m}$ is relatively large, so that the buffer layer plays a role 


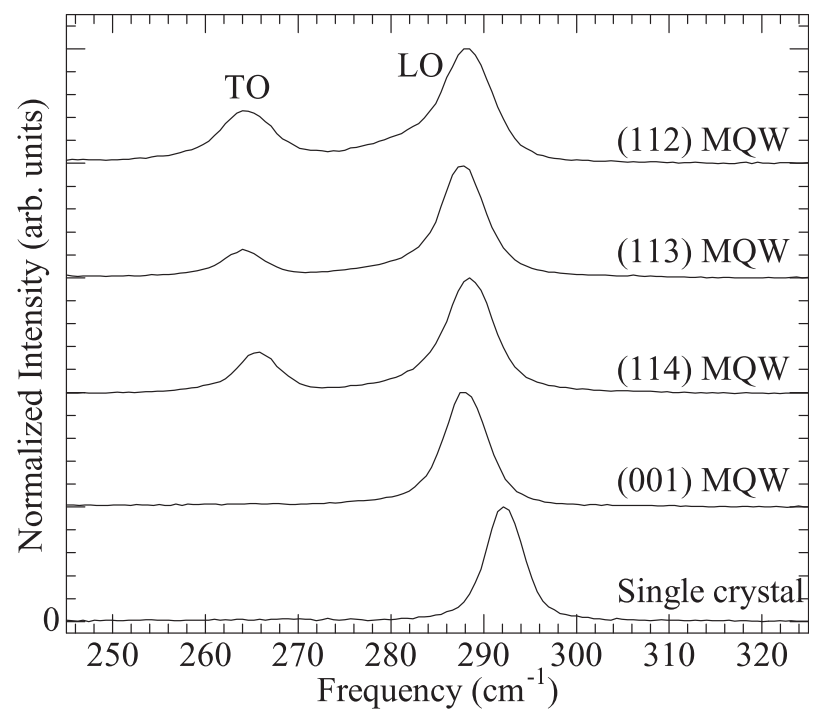

FIG. 1. Raman scattering spectra of the (001)-oriented GaAs single crystal, (001), (114), (113), and (112) MQWs at room temperature. For clarity, each spectrum is normalized at the peak intensity.

of a quasi-substrate; namely, only the GaAs layer in the MQW structure is under the biaxial stain resulting from the lattice mismatch between $\mathrm{GaAs}$ and $\mathrm{In}_{0.1} \mathrm{Al}_{0.9} \mathrm{As}$, which produces the piezoelectric field in the (112), (113), and (114)oriented GaAs layer. The MQW structure of each sample consisted of 20 periods of the GaAs and $\operatorname{In}_{0.1} \mathrm{Al}_{0.9}$ As layers. The thicknesses of the GaAs and $\operatorname{In}_{0.1} \mathrm{Al}_{0.9} \mathrm{As}$ layers were the same: $10 \mathrm{~nm}$. The biaxial tensile strain $\varepsilon$ in the GaAs layer is estimated from the lattice mismatch to be $0.84 \%$, where the lattice constants of GaAs and $\mathrm{In}_{0.1} \mathrm{Al}_{0.9} \mathrm{As}$ are 0.5633 and $0.5701 \mathrm{~nm}$, respectively. ${ }^{13}$ Hereafter, we simply call the $(11 n)$-oriented $\mathrm{GaAs} / \mathrm{In}_{0.1} \mathrm{Al}_{0.9} \mathrm{As}$ strained MQW as the $(11 n)$ MQW. All of optical measurements were performed at room temperature.

In advance to the measurement of terahertz waves, we measured the Raman scattering spectra of the present samples and of a reference sample that is a (001)-oriented GaAs single crystal. The photon energy and power of the excitation beam were $2.33 \mathrm{eV}$ and $1.3 \mathrm{~mW}$, respectively. The diameter of the beam spot on the sample surface was about $10 \mu \mathrm{m}$. Figure 1 shows the Raman spectra in the frequency range of the GaAs optical phonons. In the GaAs bulk crystal, the Raman spectrum shows the band peaking at the frequency of $292 \mathrm{~cm}^{-1}$. This band is attributed to the GaAs LO phonon. ${ }^{14}$ We note that the peak frequencies of the GaAs-like LO phonon bands in the $(11 n) \mathrm{MQWs}$ are shifted by $-4 \mathrm{~cm}^{-1}$. According to Ref. 15 , the strain-induced frequency shift, $\Delta \omega$, of the GaAs-like LO phonon is in the following relation with $\varepsilon: \Delta \omega$ $\approx-3.8 \times 10^{2} \varepsilon\left(\mathrm{cm}^{-1}\right)$. The value of $\varepsilon$ is estimated from the phonon-frequency shift to be $1.0 \%$ that is almost in agreement with the estimated value described above. In addition, the transverse optical (TO) phonons are observed at $264 \mathrm{~cm}^{-1}$ in the (112), (113), and (114) MQWs. Since the TO-phonon mode is allowed in (111)-oriented GaAs, it is evident that the crystal orientation of the (11n) MQW actually approaches the (111) orientation. Accordingly, the abovementioned facts suggest the presence of piezoelectric fields in the GaAs layers of the (112), (113), and (114) MWQs. We

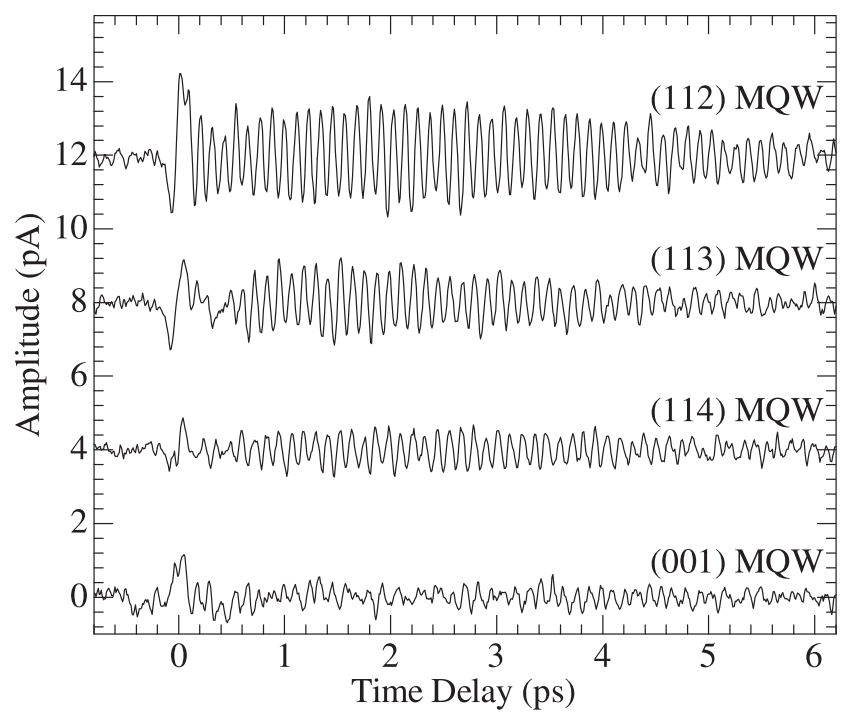

FIG. 2. Amplitudes of the terahertz waveforms of the (001), (114), (113), and (112) MQWs as a function of time delay at room temperature. For clarity, each waveform is vertically shifted.

note that the frequencies of the LO and TO phonons do not depend on the crystal orientation of the samples. This is consistent with the fact that the stain-induced frequency shifts of the optical phonons of GaAs in the [001] and [111] directions are almost the same. ${ }^{16}$ The piezoelectric fields in the GaAs layers of the (112), (113), and (114) MQWs are estimated from $\varepsilon$ to be 41,72 , and $144 \mathrm{kV} / \mathrm{cm}$, respectively, according to the theory in Ref. 17. In the (001) MQW, the piezoelectric field is not produced because the tetragonal biaxial strain in the (001)-oriented GaAs layer does not have the ability to induce the piezoelectric field. ${ }^{17}$ As shown in the calculated results, the piezoelectric field increases as the orientation index $n$ approaches 1 . This is because the GaAs crystal has a polar axis along the [111] direction.

Next, we performed the terahertz-wave measurement under the condition of dry $\mathrm{N}_{2}$ purge, using a Ti:sapphire femtosecond pulse laser. The duration time and repetition of the laser pulses were about $50 \mathrm{fs}$ and $90 \mathrm{MHz}$, respectively. The pump beam was focused on the sample with the incidence angle of $45^{\circ}$. The diameter of the beam spot on the sample surface was about $100 \mu \mathrm{m}$. The emitted terahertz wave was collected with use of two off-axis parabolic mirrors, and was detected by an optically gated dipole antenna with a gap of $6.0 \mu \mathrm{m}$ formed on a low-temperature-grown GaAs layer. The powers of the pump and gate beams were fixed to $100 \mathrm{~mW}$ and $10 \mathrm{~mW}$, respectively. The photon energies of both the beams were the same value of $1.55 \mathrm{eV}$. The terahertz waveform of each (11n) MQW is shown in Fig. 2. The monocycle signal, which originates from the surge current of photogenerated carriers, ${ }^{18}$ appears around the time delay of $0 \mathrm{ps}$. The monocycle signal is followed by the relatively long oscillation pattern with a period of $115 \mathrm{fs}$. We emphasize that the amplitude of the oscillation pattern is enhanced as the orientation index $n$ approaches 1 .

In order to analyze the oscillation pattern, we applied the Fourier transform to the terahertz waveform. Figure 3 shows the Fourier power spectrum of each MQW. The weak signals in the frequency range from 0 to $5 \mathrm{THz}$ correspond to 


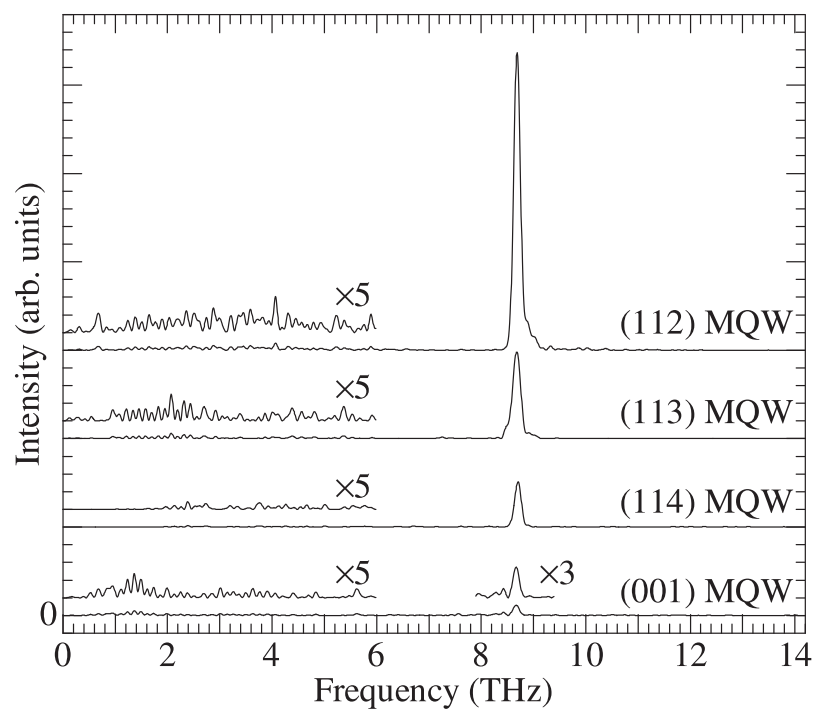

FIG. 3. Fourier power spectra of the terahertz waveforms shown in Fig. 2.

the monocycle signals of the terahertz waveforms around 0 ps. The noteworthy phenomenon appears in the frequency range from 8 to $9 \mathrm{THz}$. The intense terahertz bands have a peak at $8.7 \mathrm{THz}$ in the (114), (113), and (112) MQWs. Taking account of the fact that the frequency of the GaAs-like LO phonons in the $(11 n)$ MQWs is $288 \mathrm{~cm}^{-1}(=8.64 \mathrm{THz})$ as shown in Fig. 1, the terahertz band peaking at $8.7 \mathrm{THz}$ is attributed to the coherent GaAs-like LO phonon. The intensity of the coherent GaAs-like LO phonon band markedly increases as the orientation index $n$ approaches 1. As mentioned in the results of the Raman measurement, the piezoelectric field is enhanced as the orientation index $n$ approaches 1 because the GaAs crystal has the polar axis along the [111] direction. It is, therefore, concluded that the intensity of the terahertz band of the coherent GaAs-like LO phonon is enhanced by the piezoelectric field.

Finally, we discuss the relation between the terahertzband intensity of the coherent GaAs-like LO phonon and piezoelectric field. It is well known that the coherent LO-phonon amplitude is proportional to an electric field. ${ }^{12}$ Taking account of this fact, as shown in Fig. 4, we plot the square root of the Fourier power intensity of the coherent GaAs-like LO-phonon band (solid circle), which corresponds to the amplitude, as a function of estimated piezoelectric field. We note that the line widths, the full widths at half maximum, of the coherent GaAs-like LO-phonon bands are the same value of $0.12 \mathrm{THz}$ in all of the samples. The line width corresponds to the phonon relaxation time, so that the phonon relaxation times are the same in all of the samples. This means that it is reasonable to directly compare the values of the square root of the Fourier power intensity among the samples. The solid line is the fitted result using a linear function. The fitted result well explains the experimental results; namely, the amplitude of the coherent GaAs-like LO phonon is actually proportional to the piezoelectric field. This means that the initial polarization, which is produced by the piezoelectric field, dominates the amplitude of the terahertz wave from the coherent GaAs-like LO phonon. The fitted line has an offset value at $0 \mathrm{kV} / \mathrm{cm}$ of the piezoelectric

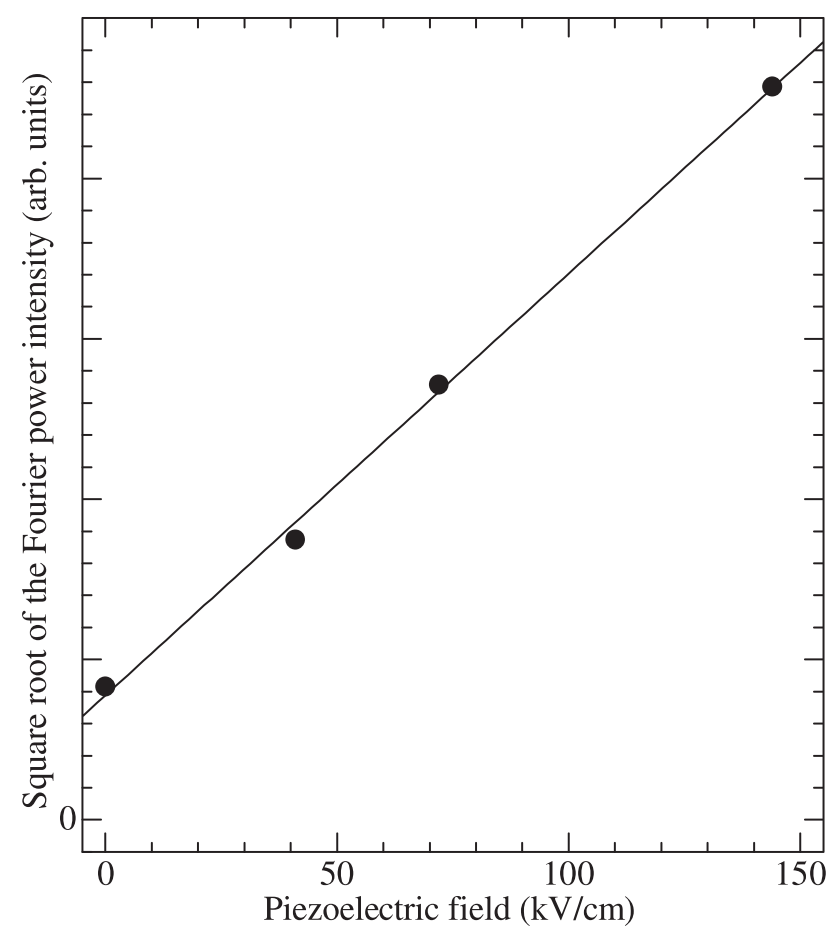

FIG. 4. Square root of the Fourier power intensity of the coherent GaAslike LO-phonon from the $(11 n)$ MQWs plotted as a function of piezoelectric field. The solid circle corresponds to the experimental result, while the solid line is the fitted result with use of a linear function.

field. This offset originates from the intrinsic surface built-in potential in the (001) MQW.

In summary, we have systematically investigated the terahertz wave from the coherent LO phonon in the $(11 n)$ oriented $\mathrm{GaAs} / \mathrm{In}_{0.1} \mathrm{Al}_{0.9} \mathrm{As}$ strained MQWs with $n=2,3,4$, and $\infty$. We estimated the biaxial tensile strain from the frequency shift of the GaAs-like LO phonon, using the Raman scattering measurement. We also estimated the piezoelectric field from the strain and confirmed that the piezoelectric field is enhanced as the orientation index $n$ approaches 1 . The intensity of the terahertz band of the coherent GaAs-like LO phonon band in the $(11 n)$ MQW markedly increases as the orientation index $n$ approaches 1 . Consequently, we conclude that the piezoelectric field in the strained GaAs layer of the (11n) MQW enhances the terahertz wave from the coherent GaAs-like LO phonon. The present conclusion is supported by the fact that the amplitude of the coherent GaAs-like LO phonon is proportional to the piezoelectric field.

One of the authors, H.T., acknowledges the support of Grant-in-Aid for Young Scientists (B) No. 22760010 from the Japan Society for the Promotion of Science. The author, M. N., is thankful to Grant-in-Aid for Challenging Exploratory Research, No. 24656018, from Japan Society for the Promotion of Science.

${ }^{1}$ For a review, P. Gu and M. Tani, in Terahertz Optoelectronics, edited by K. Sakai (Springer, Berlin 2005), pp.63-97, and relevant references therein.

${ }^{2}$ T. Dekorsy, H. Auer, C. Waschke, H. J. Bakker, H. G. Roskos, H. Kurtz, V. Wagner, and P. Grosse, Phys. Rev. Lett. 74, 738 (1995).

${ }^{3}$ M. Tani, R. Fukasawa, H. Abe, S. Matsuura, K. Sakai, and S. Nakashima, J. Appl. Phys. 83, 2473 (1998).

${ }^{4}$ R. Kersting, J. H. Heyman, G. Strasser, and K. Unterrainer, Phys. Rev. B 58, 4553 (1998).

${ }^{5}$ A. Leitenstorfer, S. Hunsche, J. Shah, M. C. Nuss, and W. H. Knox, Phys. Rev. Lett. 82, 2473 (1999). 
${ }^{6}$ P. Gu, M. Tani, K. Sakai, and T.-R. Yang, Appl. Phys. Lett. 77, 1798 (2000).

${ }^{7}$ M. P. Hasselbeck, D. Stalnakaer, L. A. Schlie, T. J. Rotter, A. Stinz, and M. Sheik-Bahae, Phys. Rev. B 65, 233203 (2002).

${ }^{8}$ Y. C. Shen, P. C. Upadhya, E. H. Linfield, H. E. Beere, and A. G. Davis, Phys. Rev. B 69, 235325 (2004).

${ }^{9}$ K. Mizoguchi, T. Furuichi, O. Kojima, M. Nakayama, S. Saito, A. Syouji, and K. Sakai, Appl. Phys. Lett. 87, 093102 (2005).

${ }^{10}$ K. Mizoguchi, A. Mizumoto, M. Nakayama, S. Saito, A. Syouji, N. Yamamoto, and K. Akahane, J. Appl. Phys. 100, 10357 (2006).

${ }^{11}$ M. Nakayama, S. Ito, K. Mizoguchi, S. Saito, and K. Sakai, Appl. Phys. Express 1, 012004 (2008).
${ }^{12}$ T. Pfeifer, T. Dekorsy, W. Kütt, and H. Kurtz, Appl. Phys. A 55, 482 (1992).

${ }^{13}$ Semiconductors-Basic Data, edited by O. Madelung (Springer, Berlin 1996). The lattice constant of $\operatorname{In}_{0.1} \mathrm{Al}_{0.9}$ As is estimated with use of a linear interpolation between those of InAs and AlAs.

${ }^{14} \mathrm{~N}$. Jusserand and M. Cardona, in Light Scattering in Solids V, edited by M. Cardona and G. Güntherodt (Springer, Berlin, 1989), Chap. 3.

${ }^{15}$ M. Nakayama, K. Kubota, T. Kanata, H. Kato, S. Chika, and N. Sano, J. Appl. Phys. 58, 4342 (1985).

${ }^{16}$ F. Cerdeira, C. J. Buchenauer, F. H. Pollak, and M. Cardona, Phys. Rev. B 5, 580 (1972)

${ }^{17}$ E. Anastassakis, Phys. Rev. B 46, 4744 (1992).

${ }^{18}$ X. -C. Zhang and D. H. Auston, J. Appl. Phys. 71, 326 (1992). 